\title{
Nuclear Project: Preliminar Study of the Hydrogen Flux in Palladium Film under Electric Field
}

\author{
De Ninno A. ${ }^{1}$, Bassignana A. ${ }^{2}$, Musumeci F. ${ }^{3,4}$, Tudisco S. ${ }^{4}$ and Cammarata G. ${ }^{\text {* }}$ \\ ${ }^{1}$ ENEA, FSN-TECFIS C.R. Frascati (Roma) \\ ${ }^{2}$ Consultant, Bergamo \\ ${ }^{3}$ DFA- Università di Catania via S. Sofia 64, 95123 Catania \\ ${ }^{4}$ INFN-LNS via S. Sofia 62, 95123 Catania \\ ${ }^{5}$ DII- Università di Catania via S. Sofia 64, 95123 Catania \\ Email: cammaratagiuliano@gmail.com
}

\begin{abstract}
NUCLEAR (Nuclear CLEAn Reactor) Project arises from a scientific collaboration among of the Department of Industrial Engineering and the Department of Physics and Astronomy of the University of Catania, and the TECFIS Division of the Frascati Research Center of ENEA. Aim of the project is to develop a device prototype able to check the production of clean energy trough Lattice Assisted Nuclear Reactions LANR.

The first step of the project is to study an engineered version of an experiment realized at ENEA Frascati in 1992 by one of the authors (ADN). It has been shown that whenever a flux of deuterons crosses a strong deuterated palladium membrane, an anomalous excess heat is produced which cannot be accounted for conventional chemical reactions. That experiment was realized in electrolysis of heavy water in order to produce a flux of $\mathrm{d}^{+}$towards a cathode made out of 500 microns thick palladium membrane. The thermal anomaly has been shown to be related to the flux of atoms crossing the metal. The new design of the experiment avoids the obstacle of electrolysis such as the poisoning of the materials, due to long time running, using Polymer Electrolyte Membranes (PEM) such as those used in fuel cells. Furthermore is possible to drive the current of positive ions applying an electric field across the PEM. Preliminary measures have shown that the flux obtained in such a configuration is even greater than the flux obtained in electrolysis with several hundreds of milliampere and it is linearly related to the voltage across the PEM. The condition of the experiments thus result to be easily and accurately controlled and reproducible.
\end{abstract}

Keywords: Lattice Assisted Nuclear Reaction, Palladium film, PEM.

\section{INTRODUCTION}

A broad variety of experiments have been proposed in the last 25 years in order to replicate the excess heath shown by Fleischmann and Pons in 1989 in their electrolytic cell [1]. The main obstacle to the acceptance by the scientific community of the research of the heath production in lattice assisted nuclear reactions (LANR) even known as low energy nuclear reactions (LENR) is out of doubt the difficulty in having reproducible experiments. The excess heath has been mainly observed in highly hydrated or deuterated metals such as Palladium or Nickel often exposed to treatment aimed to induce a thermal or a mechanical stress. A detailed discussion of these techniques is out of the scope of this paper, however, is it here important to underline which, in our view, are the main steps of all the successful experiments performed up to now: 1) the loading step; 2) the obtainment of a stationary high loading phase in the lattice; 3 ) rapid raise of gradients of hydrogen (deuterium) concentration in the host lattice.
Electrolysis is a promising solution for hydrogen production from renewable resources. The reaction of splitting water into hydrogen and oxygen takes place in a cell consisting of an anode and a cathode separated by an electrolyte. Hydrogen ions $\mathrm{h}^{+}$migrate towards the cathode and, depending on the nature of its material, can be loaded into its lattice and diffuse through its thickness. Hydrogen can be loaded into materials even by exposure to the gas phase, pressure and temperature of the gas depending on the material.

$\mathrm{Pd} / \mathrm{H}(\mathrm{D})$ system has been the first that had attracted research activities [2] because it represents one of the most transparent and instructive models for a metal-hydrogen system. It has been well established that hydrogen can be loaded in the palladium lattice even above the loading ratio $\mathrm{x} \approx 0.8$ (atoms of hydrogen per atom of $\mathrm{Pd}$ ). Palladium hydride shows many interesting features, most of them not explained yet, among the others it is worthwhile to underline the following:

$\checkmark \quad$ The dissolved hydrogens get ionized when entering 
into the lattice [3]

$\checkmark \quad$ The Pd-H system shows a complex phase diagram: at very low concentration $\mathrm{x} \leq 0.05$ (phase) hydrogen is in gaslike situation within the lattice; increasing the concentration small island of a different phase appear (phase) where hydrogen ions are confined in the octahedral sites of $\mathrm{Pd}$, the co-existence of the and phase is allowed at room pressure and temperature in the range $0.05 \leq \mathrm{x} \leq 0.6$; at higher concentration a new phase appears (phase) where ions occupy the tetrahedral position of the lattice [4]

$\checkmark \quad$ There is the electrochemical evidence that, at high values of $\mathrm{x}$, ions perform wide oscillations in an almost unbounded state in $\mathrm{Pd}$ lattice [5] and that the diffusion coefficient $\mathrm{D}$ of the hydrogen isotopes in $\mathrm{Pd}$ is $D(d+)>D(h+)>D(t+)$ at the contrary of what expected [6]. Since $d+$ is a boson whereas $h+$ and $t+$ are fermions, the above result hints at the existence of some sort of collective behavior.

The state of hydrogens in palladium at high concentrations bring to mind a solid state plasma made out of protons (or deuterons, or tritons) almost delocalized. This is eventually the circumstance which makes crucial the step 2 of the aforementioned procedure, i.e. the obtainment of a stable high concentration loading.

A peculiar geometry of electrolysis was set up in 1992 an experiment performed at ENEA Frascati in order to study the permeation of deuterium trough a palladium cathode [7]. The cathode was immersed by one side in the electrolytic solution, while the other side bordered with a vacuum-tight region containing deuterium gas at (about) atmospheric pressure. That experiment confirmed the production of excess heat in an electrolytic cell operating with heavy water and palladium cathode and showed, for the first time, a clear quantitative relationship between the flux of deuterium trough the cathode and the heat released. This correlation has been also unexpectedly found by a NASA researcher in 1989 [8] and, later on verified by a Chinese researcher in 2003 [9]. However, these experiments failed in obtaining a controlled process.

In the ENEA experiment, after a first phase of several hours during which the cathode lattice was loaded by the incoming deuterium, a steady flux of deuterium gas was observed in the gas chamber backward. Excess heat was measured only when two circumstances occurred: after a period in which the flux durably exceeded $10^{14}$ atoms/sec the pressure in the chamber had a sudden drop. Such an observation lead us to conclude that we had obtained somehow the formation of an high concentration gradient inside the $\mathrm{Pd}$ cathode. However, the long duration of the experiments and the high currents used, caused the poisoning of the cathode surface, due to the continuous deposition of the materials contained both in the solution and in the walls of the electrolytic cell. Even the attempt to use a palladium anode in order to regenerate the cathode surface with a continuous deposition didn't let to stabilize the experimental condition making the experiment quite difficult to be controlled. This experimental line was abandoned because of the high variability of the results and the very long time needed to obtain the right conditions.

The technological development of the Polymer Electrolyte Membrane (PEM) electrolysis, introduced to improve the industrial production of hydrogen to be used as an energy carrier, gave us the possibility to update that experiment in order to solve its major deficiencies:

a) the liquid electrolyte has been replaced by a Nafion membrane,

b) the whole cell is a "dry" cell where hydrogen/deuterium gas can flow continuously across a palladium membrane, c) the hydrogen flux is driven by an electric field applied throughout the PEM.

A long term and challenging project has been undertaken among INFN and University of Catania (Dept. of Physics and Astronomy and Department of Industrial Engineering) and ENEA (Technology for Security and Safety Division) aimed to realize a prototype device able to show that clean energy can be produced via Lattice assisted Nuclear Reactions (LANR). The project has been named NUCLEAR: Nuclear CLEAn Reactor. The first goal of this project is to realize a sealed, self-contained device able to produce a controlled, continuous flux of hydrogen/deuterium across a metallic hydride membrane. Further steps will be implemented in order to check possible thermal anomalies and/or energetic particles emission as suggested in the quoted literature.

\section{MATERIALS AND METHODS}

During the isothermal loading process, the solute gradient inside the metal suffers from a thermodynamic force influenced by all the fields that can affect the free energy of the solute.

The diffusion flux is described by the general equation:

$l=-M c \cdot \operatorname{grad} \mu$

where $c$ is the hydrogen concentration, $M$ the mobility related to $D$, the diffusion coefficient, by the Einstein relation and $\mu$ is the chemical potential. Beside the concentration gradient, the flux of solute is also affected by the elastic energy gathered into the lattice due to the deformation of the lattice parameter. The stress induced by the hydrogen diffusive flux causes an inhomogeneous distribution of the solute which can stop the diffusion of hydrogen caused by the concentration gradient, in fact the previous equation is modified as follows:

$J=-D\left(\frac{\partial c}{\partial x}-\frac{c V}{R T} \nabla \sigma\right)$

where $\mathrm{V}$ is the molar volume of hydrogen in the metal and $\sigma$ the stress field.

During the loading lattice expanded $\beta$ areas grow near $\alpha$ areas. In this region of coexistence very strong deformation fields are generated at the border between the two phases, due to the different lattice parameters. This process is highly irreversible because the energy is dissipated in the generation of dislocations. Calculations performed for a cylindrical Pd cathode show that the maximum shear stress is obtained on the surface at the beginning of the diffusion process. This means that the loading is abruptly limited to a thin slab underneath the surface, as a consequence, the loading path is very important to determine the final concentration profile inside the metal [10]. Such a problem accounts for the difficulty in obtaining high and reproducibly loading ratios. In case of hydrogen (deuterium) ions in Pd the diffusion flux gains one more term:

$J=-D\left(\frac{\partial c}{\partial x}-\frac{c V}{R T} \nabla \sigma-\frac{F z^{*} E}{R T} c\right)$ 
the last term being the electro-transport due to the action of an electric field $\mathrm{E}$ on a charge $\mathrm{z}^{*}$. An interesting evaluation of the charge $z^{*}$ has been done by measuring the Hall effect in a $\mathrm{Pd}-\mathrm{H}$ system. At about $\mathrm{x}=0.85$, each hydrogen entering the $\mathrm{Pd}$ lattice (in the $\beta$ phase) contributes about 0.75 electrons to the conduction band, whereas, for $\mathrm{x}$ exceeding that threshold, each entering hydrogen contributes more than 4 electrons to the conduction band; since hydrogen only has one electron on its own it must persuade more than three Pd electrons to jump into the higher energy band, so that the entering of one hydrogen implies a local reshuffling of the Pd structures [11].

In order to exploit electrodiffusion to manage the hydrogen flux inside $\mathrm{Pd}$ lattice, we realized a cell containing a three layer core made out of a Pt foil-Nafion membrane-Pd foil. The first Pd foil acts as ionizer allowing the hydrogen atoms to loose their electron and diffuse as a protons. A voltage was applied between the two Pd foils driving protons towards the cathode (fig. 1). The specific conductivity of the Nafion membrane is highly influenced by its water content. In case the membrane is correctly hydrated the diffusion coefficient of protons has the same order of magnitude of the diffusion coefficient in palladium. Aim of the setup was to obtain a flux of protons impinging on a target represented by the hydrated foil of palladium in order to replicate the experimental condition of the experiment quoted in [7] bypassing the obstacle due to the electrolysis. The sandwich is hosted in a two valves Teflon cell equipped with two thermocouples to measure the temperatures in the two semi cells.

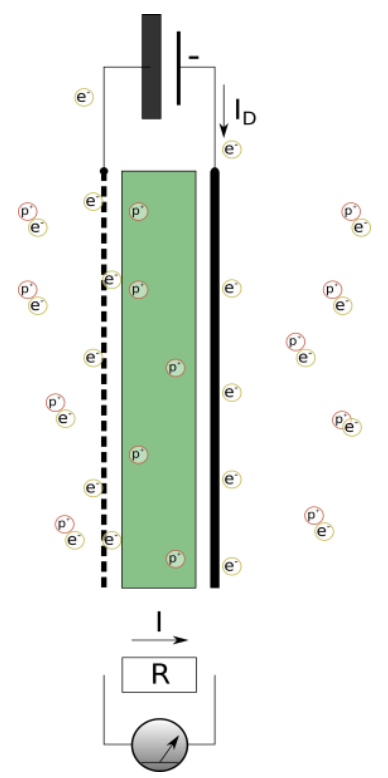

Figure 1. Schematic draw of the proton flux across the cell.

The cell also supports the use of electric heaters in order to operate at different temperatures, see Fig. 2. At the beginning of the experiment the cell is evacuated and the gas contained in a sample volume of $50 \mathrm{cc}$ is admitted to the upper part of the cell and hydrogen starts to flow across the membrane and permeates in the lower camera.

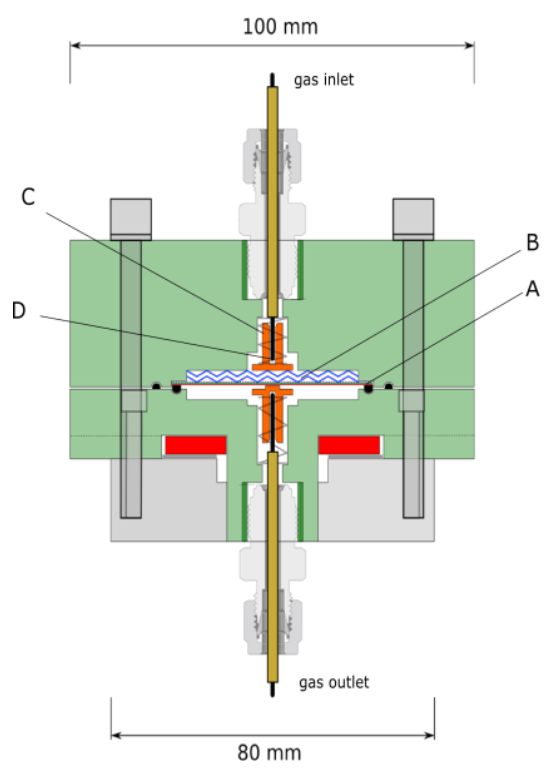

Figure 2. The experimental cell. A: Pd-Nafion-Pd membrane; B: stainless steel net used to compress the sandwich; C: Copper heather; D: thermocouple.

According to (1) the flux (mbar/s) across the cell depends on the applied voltage and on the temperature, see Fig. 3.

This experimental layout shows that it is possible to operate in a "dry" condition obtaining proton fluxes comparable to those obtained in electrolysis at high current. Moreover, at fixed temperature, the flux is proportional to the applied voltage and it doesn't seem to be related either to the state of the surface or to the degradation of the material during the experiment.

This allows the control of the flux throughput at difference of what happen in electrolysis because of the continuously changing condition of the cathode surface.

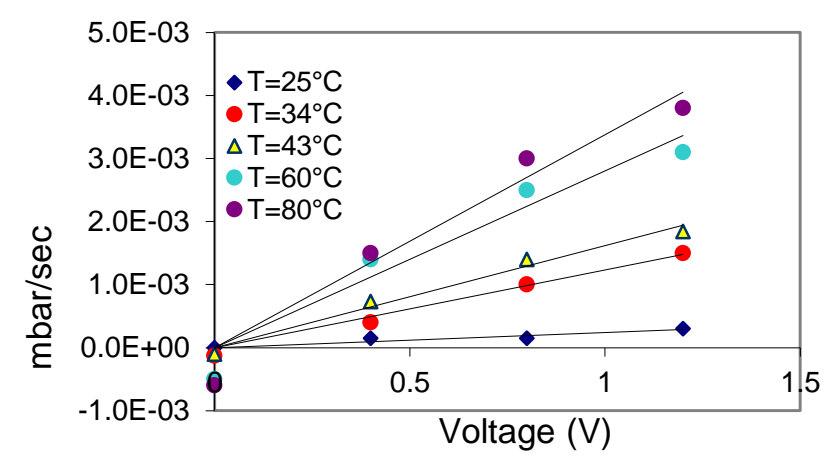

Figure 3. Measured flux

However, main technical problems have been observed with the above experimental configuration:

a) The detachment of the Pd film from the PEM due to its deformation during the formation of the hydride (see Fig. 4). It is, in fact, well known that the palladium lattice increases its lattice length up to $10 \%$ during the hydrogen uptake. The formation of a gas layer between the Pd sheet and the PEM allow the recombination of protons and electrons and the formation of the $\mathrm{H}_{2}$ and limits the overall flux across the membrane.

b) The impossibility to monitor any possible anomaly in the temperature due to the high mass of the Teflon which makes very slow the thermal response of the cell. 


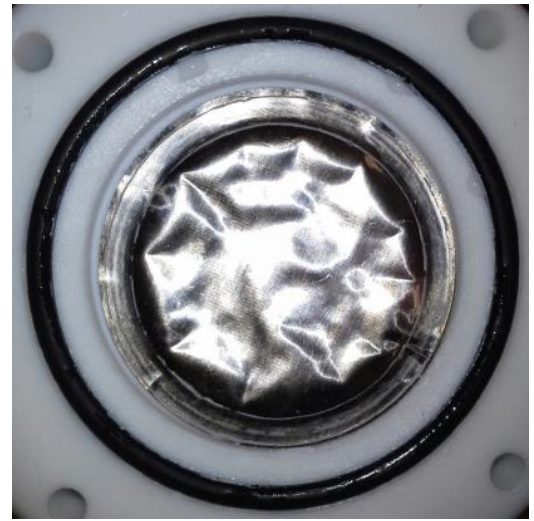

Figure 4. The Pd cathode after few hours of operation in the cell

The NUCLEAR project has been conceived just to overcome these problems: a new cell has been designed and realized reducing the overall mass. On both sides the reactor is enclosed by two symmetric flanges equipped with an optical window. One window is set with a quartz window for IR measurements while the other would host an X-ray detector. (Fig. 5).

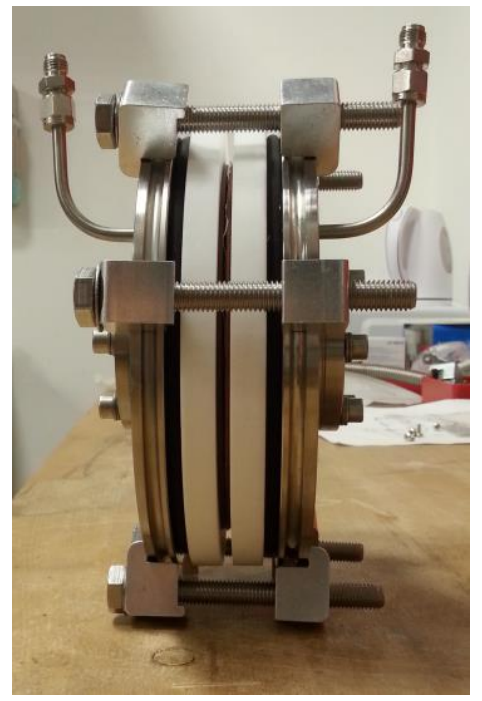

Figure 5. Lateral view of the cell

The most challenging task will be to set up a procedure to realize a compact sandwich where the electrodes (cathode and anode) are directly deposited onto the PEM in order to avoid protons-electrons recombination, and subsequent loose of energy. Beside Nafion we also plan to use ceramic proton conductors operating between 80 and $400{ }^{\circ} \mathrm{C}$.

\section{CONCLUSIONS}

The NUCLEAR Project is aimed to perform a critical review of experiments which, in the past decades, have shown the existence of thermal emission during the interaction between hydrogen (deuterium) and hydrated (deuterated) metals such as palladium or nickel. In particular, the analysis of the literature has shown that in the most of the successful experiments, the following conceptual block diagram has been followed:

a) Hydrogen (deuterium) ionization. Hydrogen (deuterium) molecule is splitted onto the surface of a catalyst such as $\mathrm{Pt}$ or $\mathrm{Pd}$ at room temperature or $\mathrm{Ni}$ at high temperature $\left(>280{ }^{\circ} \mathrm{C}\right)$ or is obtained by electrolysis of water.

b) Interaction with the lattice The atomic hydrogen (deuterium) is then loaded into a lattice where it migrates as a proton (deuteron) and form an hydride (deuteride). The loading is driven by an applied electric field. High loading ratios are obtained in suitably conditions.

c) During the process a flux of protons is assured across the target metal via a pressure or a temperature gradient.

This conceptual experiment can be realized in several way: electrolysis of (heavy) water, Cöhn-Aharonov effect [12], sputtering or co-deposition of protons (deuterons) and Pd on Pd bulk [13], high pressure flux through the hydride [14] and many others. However, the interaction of a flux of protons (deuterons) with a heavily hydride (deuteride) lattice is a basic feature of the successful experiments.

Thanks to the progress in the material science, and especially in the PEM knowledge and manufacturing, is possible to overcome many of the problems which affected the experiments in the past decades. These considerations allows to envisage a new approach to simplify the experimental design and remove the irreproducibility of the results what has been the main obstacles to the acceptance of LANR by the scientific community.

\section{REFERENCES}

[1] Fleischmann, M., Pons, S., J. Electroanal. Chem., 1989, 261, 301-308.

[2] Graham T., Phyl. Trans. Roy. Soc., 156 (1866) 415.

[3] Cöhn Z., Elektrochem, 35 (1929) 676.

[4] R.Wisniewski and J. Rostocki, Phys. Rev., B 3(2) (1971) 251.

[5] B. Dandapani, M. Fleischmann, J. Electroanal. Chem., 39 (1972) 323.

[6] Alefeld, G. Völkl, J. Hydrogen in Metals. Springer Verlag: Berlin, 1978, vol. I, pp 337-341.

[7] Bertalot L., De Marco F., De Ninno A. et al., Il Nuovo Cimento, vol. 15D, no. 11, (1993) 1435-1443.

[8] Fralick G.C. et al., Technical Memorandum, 102430, 1989.

[9] Li Xing Z., Liu B., et al. Correlation between abnormal deuterium flux and heat flow in a D/Pd system. J. Phys. D: Appl. Phys. 36 (2003) 3095-3097.

[10] R. Felici, L. Bertalot, A. De Ninno, A. La Barbera, V. Violante, Rev. Sci. Instrum. 66 (1995) 3344.

[11] Wisniewski, R., Rostocki, J. Hall Effect in the Pd-H System. Phys. Rev.1971, B3 (2), 251-252.

[12] De Ninno A., Frattolillo A., Rizzo A., Del Giudice E., Preparata G., RT/2002/41/FUS ENEA Technical Report.

[13] Szpack S., Mosier Boss P., Smith J. J., Phys. Lett A 210(6) (1996) 382-390.

[14] Arata Y., Zhang Y. J., High Temp. Soc. Jpn, 34 (2) 2008 85-93.

\section{NOMENCLATURE}

F Faraday constant, $\mathrm{Cmol}^{-1}$

$\mathrm{R} \quad$ Molar gas constant, $\mathrm{Jmol}^{-1} \mathrm{~K}^{-1}$

$\mathrm{T} \quad$ Temperature, $\mathrm{K}$ 